\title{
The effect of space vector modulation algorithm on characteristics of three-phase voltage inverter for drives of optical telescopes
}

\author{
V. TOMASOV*, A. USOLTSEV, P. ZOLOV, and P. GRIBANOV \\ ITMO University, 49 Kronverksky Pr., St. Petersburg, 197101, Russia
}

\begin{abstract}
The effect of space vector modulation algorithm on magnitude of current vector ripples was considered on the mathematical model of a three-level three-phase voltage inverter with symmetric active inductive load. Limitations on the use of such inverters as a power supply for precision drive of optical telescopes were specified.
\end{abstract}

Key words: multilevel inverter, space vector modulation, modulation algorithm, current ripples, precision AC drive.

\section{Introduction}

Voltage source inverters (VSI) are used as power supply for precision AC drives of optical telescopes. These drives must provide high pointing accuracy to space objects, moving in tracking mode with infralow velocity (about seconds of arc per second), and moving with relatively high velocity, so total control range is 15000 ... 20000:1, while the mean square error (MSE) should not exceed $1 \ldots 2$ [1-3].

Voltage inverters are impulse regulators forming the load current by stepwise changes of voltage.

It is difficult to achieve high demands of drive coordinates regulation by using two-level inverters, so increasingly often developers apply multi-level VSI, which combines capabilities of pulse-amplitude and pulse-width modulation. It allows for reducing the power switching effect on control object, to refine spectral composition of output voltage and current, and to reduce dynamic losses in inverter leading to optimizing weight and dimensions of drive power subsystem.

Space vector modulation (SVM) is successfully used as AC drives power supplies [4-15]. It allows to reduce current ripples against sinusoidal pulse width modulation (PWM), however, the problem still requires a solution for high-quality drives with wide range of speed control.

Obviously, there are two approaches to the problem: 1) increasing the number of possible states of the inverter output voltage (base vectors) and 2) improvement of the algorithm of state changing. The first way leads to the invention of multilevel inverters with different structure (half-bridge inverters with blocking diodes and a "floating" capacitor, inverters with single-phase bridges). The second - to the developing and improving algorithms of PWM.

One of the interesting algorithmic ways of reducing current ripple is a virtual increase of the base vectors number by di-

\footnotetext{
*e-mail: tomasov@ets.ifmo.ru
}

Manuscript submitted 2017-07-14, revised 2017-08-24, initially accepted for publication 2017-08-28, published in October 2017. viding the modulation period into two or three equal time intervals, within which the base vectors bounding 60-degree sector containing the modulation voltage vector are formed [16-18]. Thus, the number of base "vectors" increases to 12 and 56, accordingly. But it significantly complicates the control system.

Eventually, VSI of AC drive must form a circular hodograph of stator flux linkage, or, equivalently, of stator current, in steady state mode. The trajectory of stator current vector between adjacent commutations of inverter switches is determined by the attitude and magnitude of active voltage base vector. Therefore, different sequences or algorithms of base vectors formation within the modulation period lead to different trajectories of current vector and different ripples. The effect of the choice of sequence of base vectors forming on the current (flux linkage) ripples during transitions of modulation vector across the segment borders is considered in $[19,20]$. However, the kind and magnitude of ripples depends not only on voltage jumps at the boundaries, but also on the algorithm (sequence) of vectors forming within segments. Moreover, the number of algorithms excluding redundancy of base vectors for threephase VSI is six.

This work covers the investigation of effect of modulation algorithm on current ripples in the load of the inverter. Threelevel inverter was selected as an object of study, as it most clearly demonstrates this effect in different operation modes. At the same time, the findings may be extended to inverters with more levels, as well as to two-level inverters. Moreover, during deep control, which is needed to achieve ultra low velocities of the drive, current formation operates on two or three-level mode, regardless of the total number of levels of VSI. At the same time, rotating EMF is close to zero, and motor becomes active inductive load for the inverter. Therefore, symmetric RL-circuit was used as the load.

\section{Base vectors plane of three-phase inverter}

All possible states of three-phase inverter output voltage can be represented as vectors, ends of which are placed at the sides 
of regular hexagons with vertices which are distant from the origin of coordinates at distances equal to the magnitudes of the DC voltages generated by the inverter. The size of the set of base vectors coordinates, including zero, is determined by the number of nonzero voltage levels $L$ as:

$$
N_{V}=1+\sum_{k=1}^{k=L} 6 \cdot k
$$

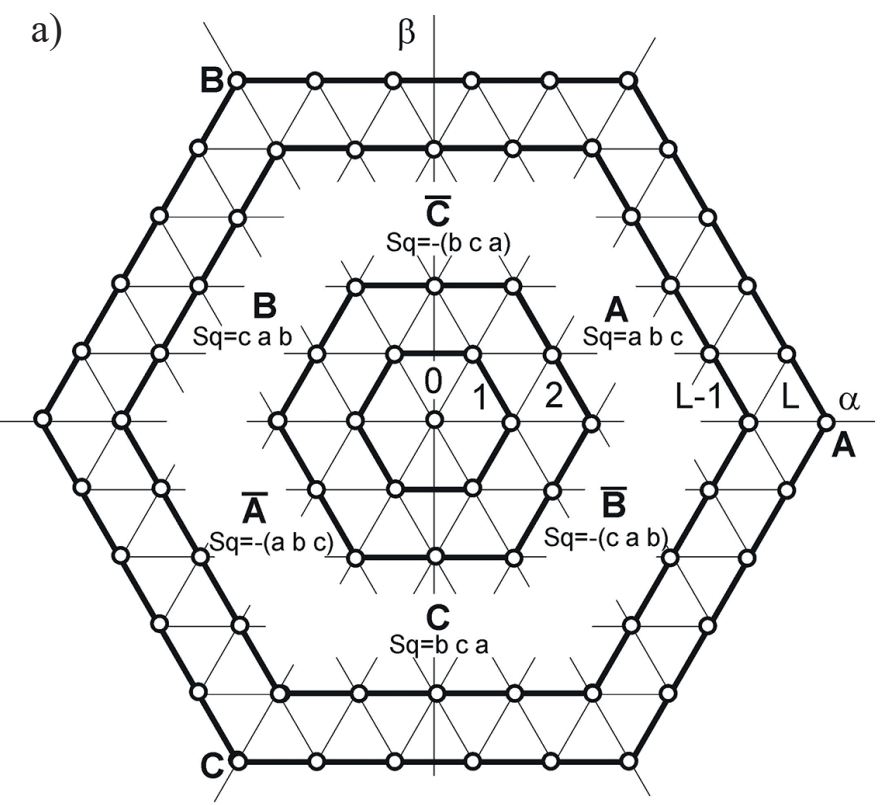

b)

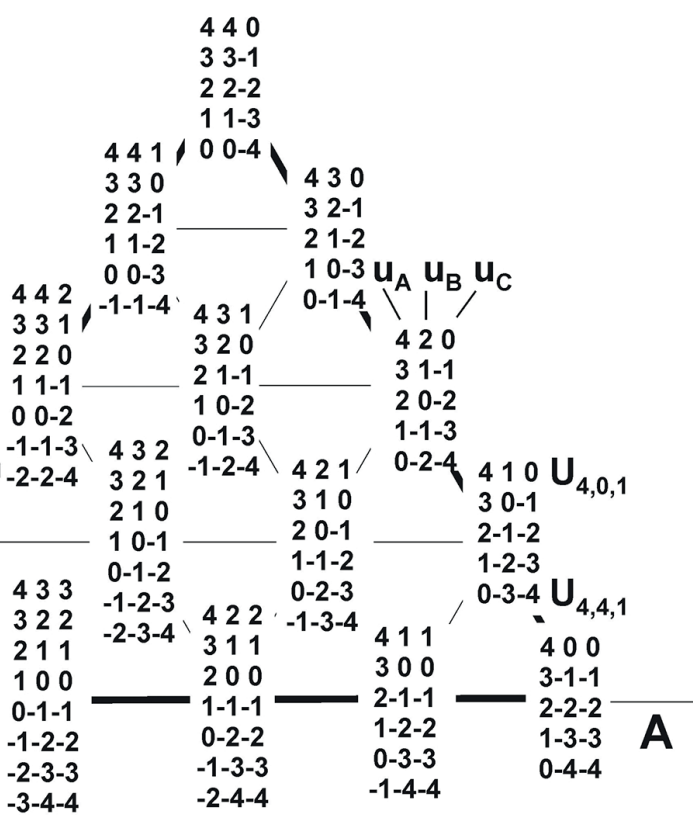

Fig. 1. Base vectors plane of L-level three-phase inverter (a) and combinations of phase voltages for sector A of the plane of five-level inverter (b)
Phase winding axes divide base vectors plane into six sectors $(A, \bar{A}, B, \bar{B}, C, \bar{C}$ in Fig. 1(a)), and ends of vectors, in turn, divide each sector into segments in form of equilateral triangles, the number of which for each $k$ zone between adjacent levels (hexagons) is equal to

$$
N_{S g k}=2 k-1
$$

where $k=1,2, \ldots L$. The total number of segments in the plane is

$$
N_{S g \Sigma}=\sum_{k=1}^{k=L} 6 N_{S g k} .
$$

Each base vector can be formed by several combinations of phase voltage levels. All possible combinations of normalized phase voltages, respective to base vectors of five-level inverter ${ }^{1}$, sector $A$, are shown in Fig. 1(b). Obviously, the number of combinations for $n$ level is

$$
N_{V n}=2 L-n+1
$$

where $n=0,1, \ldots L$

There is also a clear regularity of combinations of phase voltage levels. Let us denote by $m=0,1, \ldots N_{V n}$ the serial number of combination from the set, which corresponds to base vector $l$ from level $n$, where $l=0,1, \ldots L$ is the serial number of base vector counted from axis of phase winding $A$. Then

$$
U_{n, m, l}=\{L-m ; L-m-n+l ; L-m-n\} .
$$

Combinations of voltage levels for sector $A$ are correct for all other sectors, if circular permutations are produced as per phase sequence $S q$, shown in Fig. 1(a), in these combinations.

Redundancy of phase voltage levels combinations, forming base vectors, is used to generate switching algorithms, which optimize inverter operation in accordance with required operation mode of the load.

However, there is another way of optimizing switching algorithm, based on redundancy of base vector formation order within modulation period, i.e. forming algorithm.

\section{Determination of modulation parameters}

The principle of SVM is alternate formation during specific time intervals of three base vectors, ends of which correspond to vertices of the segment, to which modulation vector $\underline{U}^{*}=U^{*} e^{j \theta^{*}}$ (Fig. 2) belongs. Moreover, durations of formation base vectors correspond to values of oblique projections of vector $\underline{U}^{*}$ to sides of triangle of the segment, and in total is equal to modulation period $T=$ const. So modulation parameters are the number of

\footnotetext{
${ }^{1}$ the number of levels in the name of inverter corresponds to the number of nonzero positive states of phase voltages $L$ and zero value, i.e. $L+1$
} 
a)

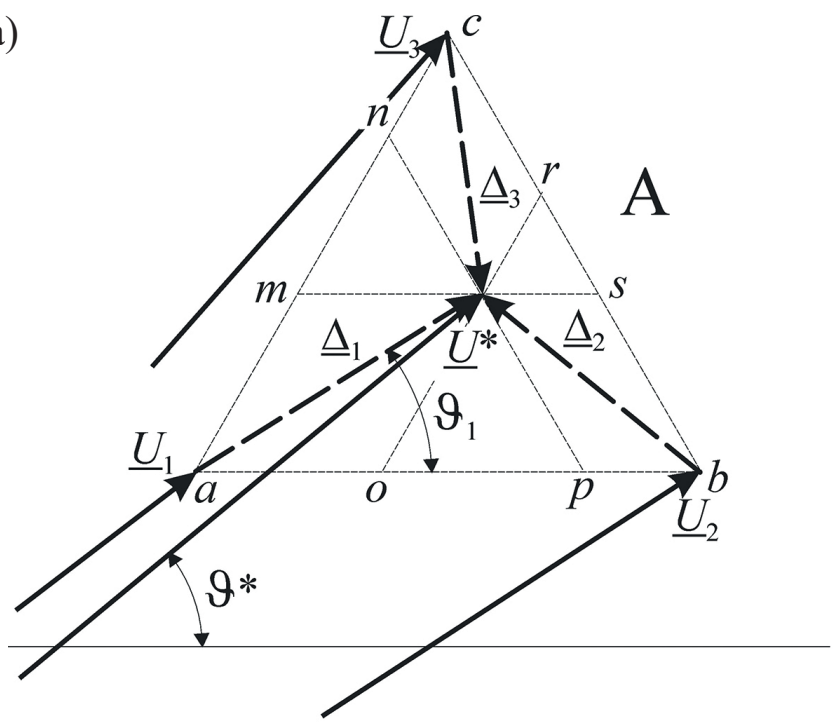

b)

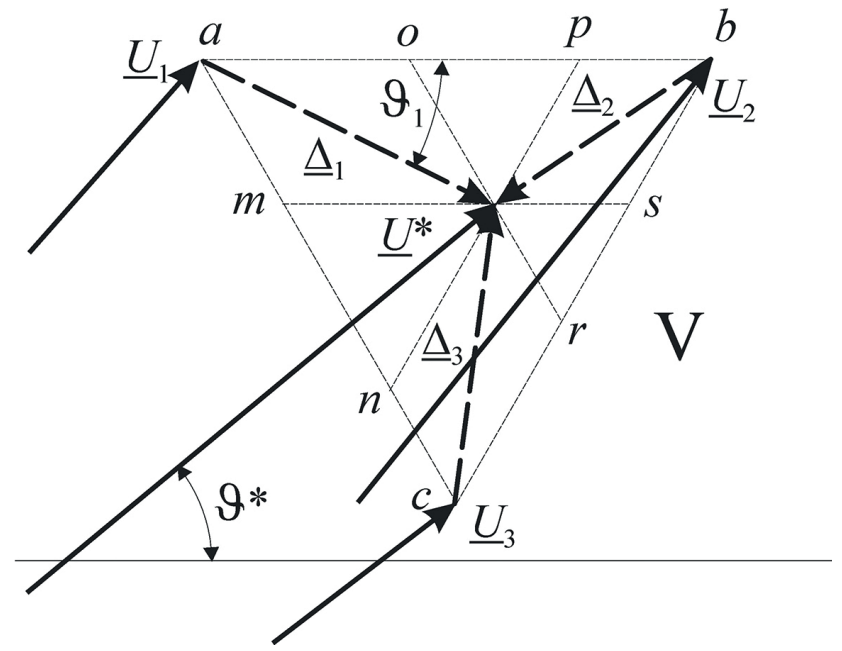

Fig. 2. Representation modulation vector $U^{*}$ as oblique projections to sides $\mathrm{A}$ and $\mathrm{V}$ of segments

segment, which determines the set of three base vectors, and durations of formation these base vectors.

Let us determine durations of forming vectors $\underline{U}_{1}, \underline{U}_{2}$ and $\underline{U}_{3}$, corresponding to Fig. 2(a)

$$
\underline{U}^{*}=\underline{U}_{1}+\underline{\Delta}_{1}=\underline{U}_{2}+\underline{\Delta}_{2}=\underline{U}_{3}+\underline{\Delta}_{3},
$$

but

$$
\begin{aligned}
\underline{\Delta}_{1} & =\underline{a m}+\underline{a o}=\left(\underline{U}_{3}-\underline{U}_{1}\right) t_{1} / T_{c}+\left(\underline{U}_{2}-\underline{U}_{1}\right) t_{2} / T_{c}= \\
& =\left[\underline{U}_{3} t_{1}+\underline{U}_{2} t_{2}-\underline{U}_{1}\left(t_{1}+t_{2}\right)\right] / T_{c}
\end{aligned}
$$

where $t_{1}$ and $t_{2}$ - durations of forming vectors $\underline{U}_{3}$ and $\underline{U}_{2}$, and $t_{3}=T_{c}-t_{1}-t_{2}$.

Then

$$
\begin{aligned}
\underline{U}^{*} & =\underline{U}_{1}+\underline{\Delta}_{1}= \\
& =\underline{U}_{1}+\left[\underline{U}_{3} t_{1}+\underline{U}_{2} t_{2}-\underline{U}_{1}\left(t_{1}+t_{2}\right)\right] / T_{c}= \\
& =\left[\underline{U}_{3} t_{1}+\underline{U}_{2} t_{2}-\underline{U}_{1}\left(T_{c}-t_{1}+t_{2}\right)\right] / T_{c}= \\
& =\left[\underline{U}_{3} t_{1}+\underline{U}_{2} t_{2}-\underline{U}_{1} t_{3}\right] / T_{c}
\end{aligned}
$$

From geometric constructions it follows that

$$
\begin{aligned}
a m & =b s=t_{1} / T_{c}=2 \Delta_{1} \sin \vartheta_{1} / \sqrt{3} \\
a o & =c r=t_{2} / T_{c}=\Delta_{1} \cos \vartheta_{1}-a m \cdot \sin 30^{\circ}= \\
& =\Delta_{1} \cos \vartheta_{1}-t_{1} /\left(2 T_{c}\right) \\
b p & =c n=t_{3} / T_{c}=1-\left(t_{1}+t_{2}\right) / T_{c} .
\end{aligned}
$$

These equations allow to determine vectors $\underline{\Delta}_{2}=b p+\underline{b s}$, $\underline{\Delta}_{3}=\underline{c n}+\underline{c r}$ and to verify that equation (8) is valid regard- less of which difference is used to calculate the vector $\underline{U}^{*}$ in (6). Typically, axis of real numbers $(\alpha)$ is superposed with axis of phase winding A, and (9) is used to calculate durations $t_{1}$, $t_{2}$ and $t_{3}$.

Adjacent segments of base vectors plane are differently orientated and relative to the axis $\alpha$; they can be called A-segments and $\mathrm{V}$-segments by appearance. If coordinate system and notations, which were used to calculate interval durations for A-segment (Fig. 2(b)), will also be used for V-segment, then (9) will be valid under the condition that the angle $\vartheta=\left|\vartheta_{1}\right|$.

The second problem to solve during the development of multilevel inverter control device is determining the segment, to which vector $\underline{U}^{*}$ belongs (Fig. 3(a)) in sector A (Fig. 1(a)). Let us call the sector, which is located between axis $\alpha$ and the beam, constituting with it an angle of $60^{\circ}$, the base sector. Oblique projections of the vector $\underline{U}^{*}$ to sides of base sector can be used to determine the segment. Indeed, projections $n$ and $m$ to sides $O L$ and $L M$ can be determined as

$$
n=\left|U^{*}\right|\left(\cos \vartheta+\frac{\sin \vartheta^{*}}{\sqrt{3}}\right) ; \quad m=\left|U^{*}\right|\left(\frac{2 \sin \vartheta^{*}}{\sqrt{3}}\right)
$$

and projections $d n$ and $d m$ to sides of segment, to which end of vector $\underline{U}^{*}$ belongs

$$
d n=n Z-n ; \quad d m=m-\text { floor }(m)
$$

where $n Z=\operatorname{ceil}(n)$ - the number of modulation area (shaded area in Fig. 3(a)).

The function $n S=\operatorname{ceil}(m)$ allows to determine the serial number of A-segment in subset of A-segments in $n Z$ modulation area. As $\mathrm{A}$ and $\mathrm{V}$ segments are alternating, so total serial number of A-segment in $n Z$ modulation area is

$$
n S_{A}=2 \cdot n S-1 .
$$


a)

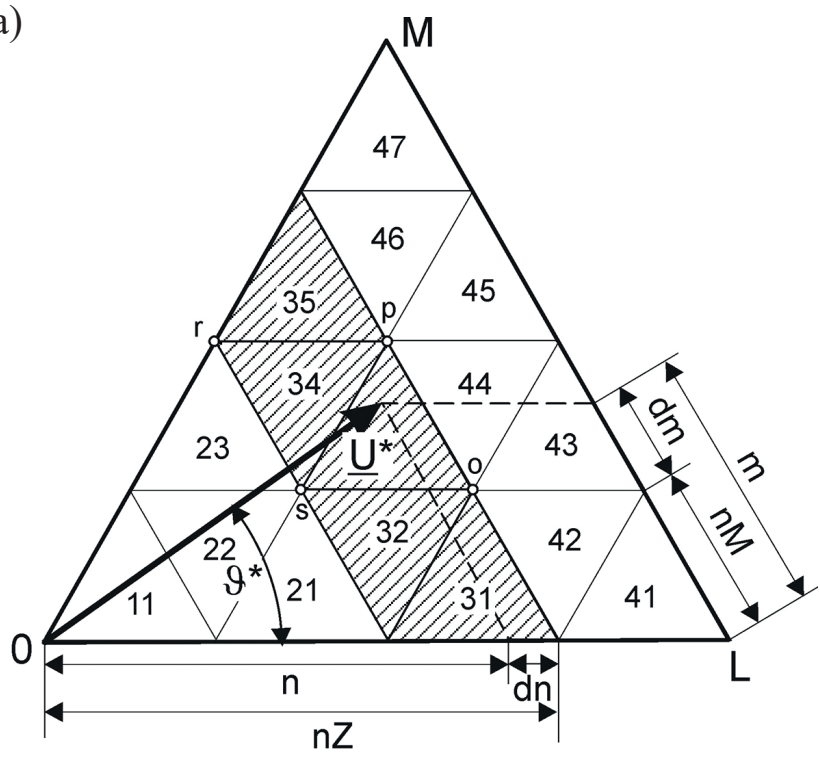

b)

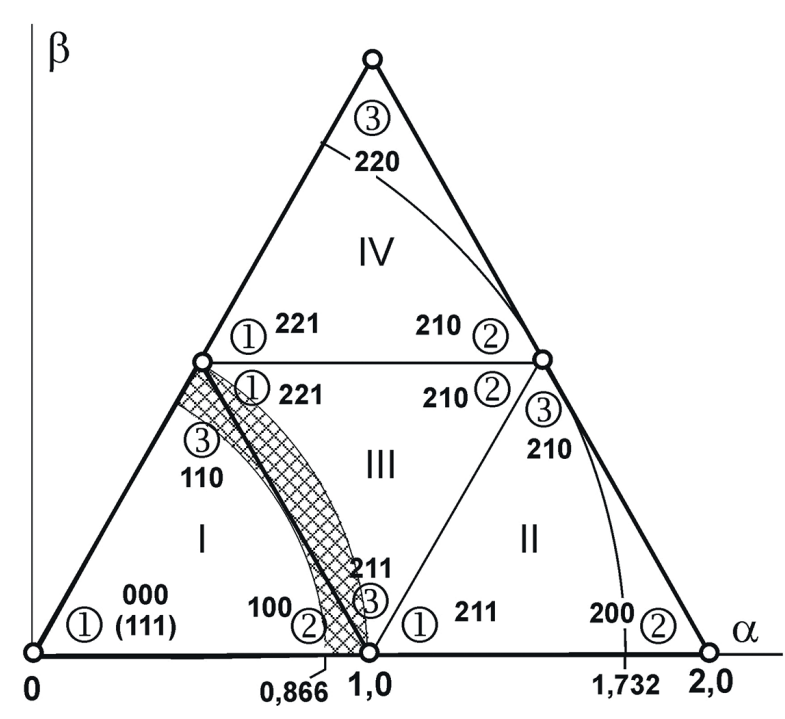

Fig. 3. Coordinates of segments in base sector five-level VSI (a) and base sector of three-level VSI (b)

One m-projection corresponds to two segments $\mathrm{A}$ and $\mathrm{V}$ in parallelogram oprs. It is possible to identify the V-segment from the condition $d n+d m>1.0$ that is easily implemented with standard rounding function $v=$ floor $(d n+d m)$.

Then the serial number of segment within full set of segments in the same modulation area is

$$
n S=n S_{A}+v
$$

For full identification of the segment, positional numeration can be used, where the most significant digit corresponds to the number of modulation area, and the less significant digit - to the number of the segment. In Fig. 3(a) such numeration is shown. Obviously, if necessary, all segments of the sector can be enumerated sequentially, as the number of segments $N_{S g k}$ is known in each $k$ modulation area. The numeration of segments allows to create the in-memory array of base vectors and fast sample as per the current location of modulation vector $\underline{U}^{*}$.

\section{SVM algorithms}

Let us consider possible modulation algorithms for three-level inverter. The base sector of such inverter is shown in Fig. 3(b), where vertices of each segment are marked with numbers in circles and three-position numbers indicate the phase voltage levels corresponding to base vectors of vertices. To reduce voltage ripples in segment of the first area (I) only single or zero voltage levels are used.

It is possible to create six algorithms of base vectors formation for segment I. In Fig. 4 these algorithms are shown for symmetric modulation with dividing two time intervals in half. The choice of one of two nonzero vectors excludes the commu- tation in one of phases of the inverter. In case of using vector $U_{0}=000$ the voltage of phase $\mathrm{C}$ remains zero value, and in case of using vector $U_{0}=111$ - the voltage of phase A stays equal to one.

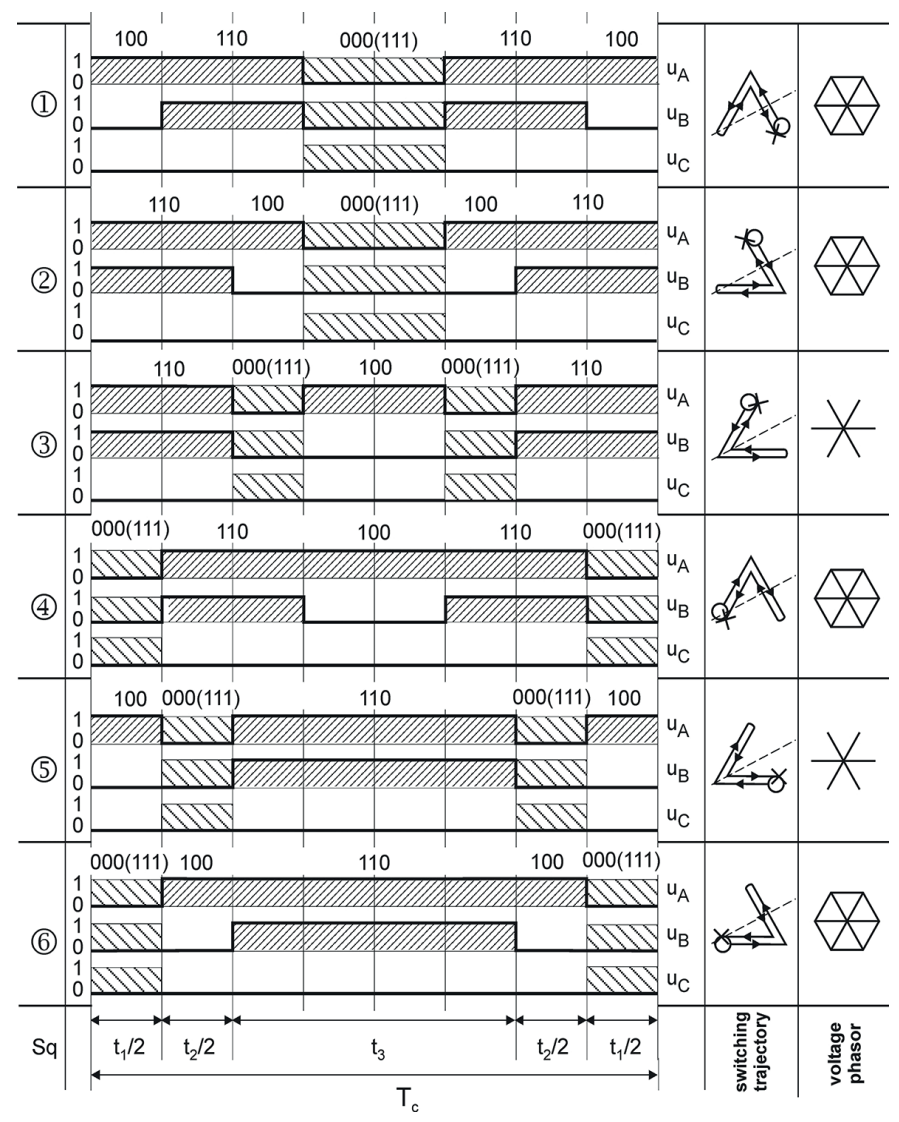

Fig. 4. Modulation algorithms for segment I 
These six algorithms form three pairs, 1-2, 3-4 and 5-6, with the same vectors generated in the middle of modulation period; they differ within pairs only by order of extreme vectors formation. Nevertheless, the form of voltage and trajectories of switching base vectors are different in all cases (Fig. 4). It should lead to different spectral distribution of voltage and different influence on the current in load of the inverter.

Similar switching functions, but with other base vectors, can be used for the rest of segments.

It should be noted that to maintain the voltage and current spectrum while changing the rotational direction of modulation vector $\underline{U}^{*}$ it is necessary to change the switching trajectory to mirror symmetric relative to the bisector of central angle of the sector (in Fig. 4, shown by dashed line). This is due to the fact that the hodograph of current vector is a response to the action of voltage vector in the given direction. Therefore, the sequence of base vectors formation in segments must be the same relative to rotational direction. If in the case of arbitrary position of vector $U^{*}$ in segment I (Fig. 3(b)) and moving in positive direction, the first forming vector of algorithm $3-2-1-2-3$ is the extreme vector in the direction of rotation (3), then for opposite rotational direction the extreme vector (2) should be formed first, and the algorithm should be 2-3-1-3-2. This condition is achieved by changing algorithms in pairs, which have an attribute of mirror symmetry of switching trajectories: 1-2, 3-5 and 4-6 (Fig. 4). Figure 5 shows the portion of current vector hodograph for all modulation algorithms and two-level operation mode of VSI for both rotational directions of vector $U^{*}$. This figure illustrates the principal asymmetry of SVM.

A feature of SVM is stepwise change of algorithm not only during transition the vector $\underline{U}^{*}$ to other sector because of its rotation, but also transition to other modulation area because of changing its magnitude.

If magnitude of modulation vector $\left|U^{*}\right|<0.866$, then threelevel inverter is working in two-level mode and has all its properties. Current in load of the inverter is forming by voltages of the first and zero levels. Let us call this area the first area of modulation. In area $0.866<\left|\underline{U}^{*}\right|<1.0$ modulation is performed by base vectors of segments I and III, i.e. voltages of all three levels. Let us call this area the transition area of mod- positive
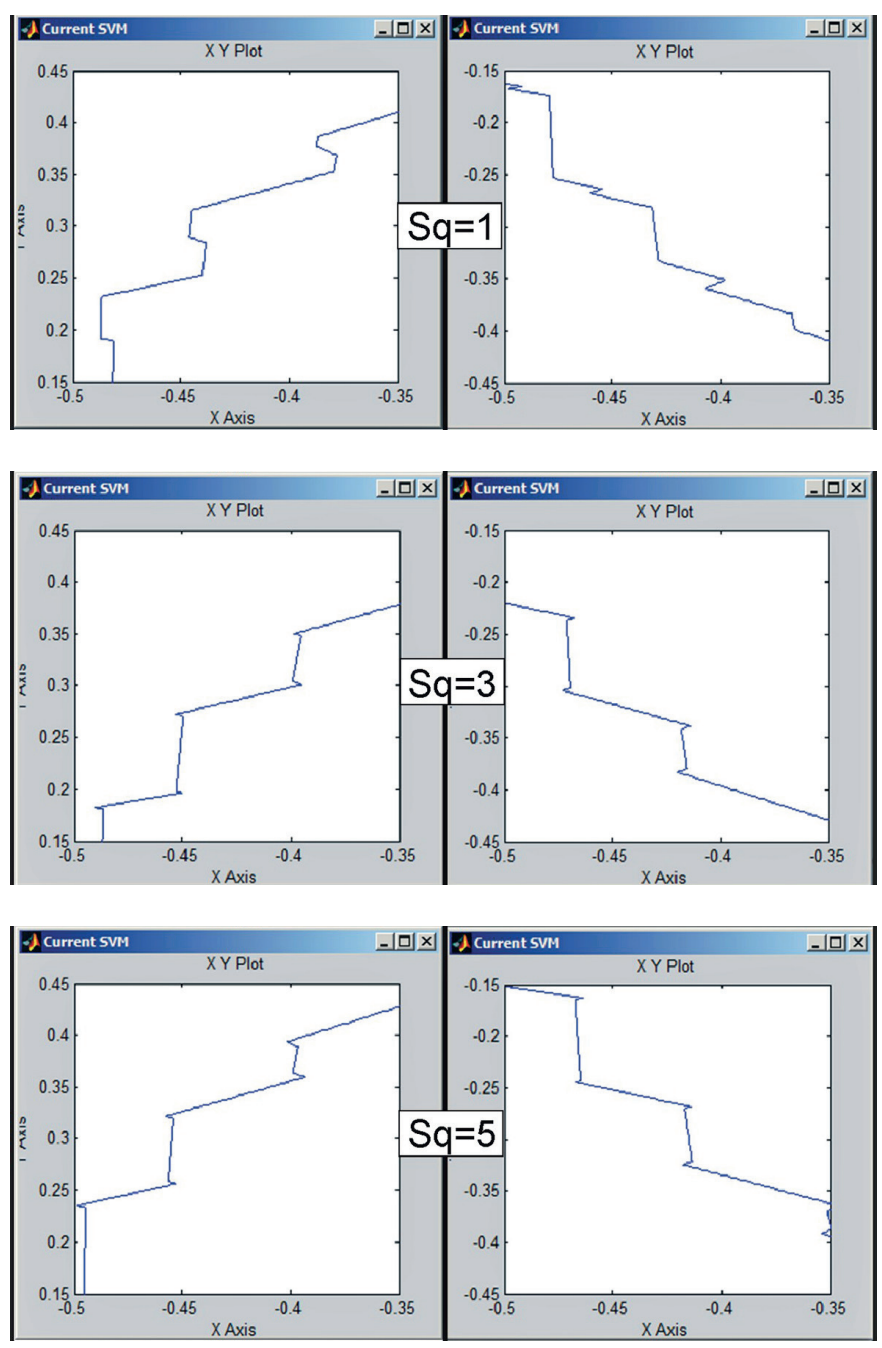

positive
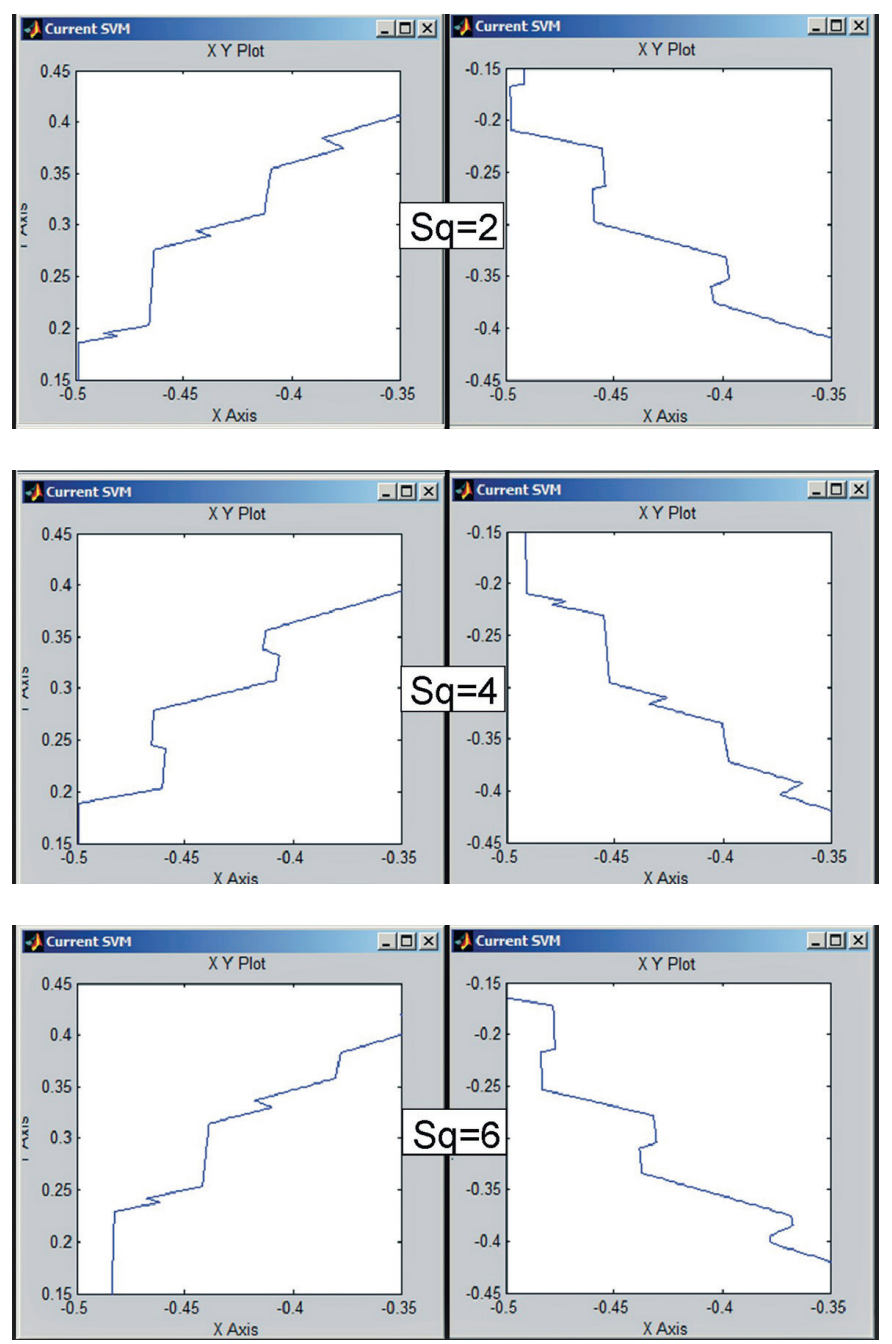

Fig. 5. Hodograph of current vector for positive and negative rotational direction of vector $\underline{U}^{*}$ 
ulation, because in next area $1.0<\left|U^{*}\right|<1.732$ output voltage is formed by base vectors of sectors II...IV only, which do not contain zero base vector and therefore can be called the second area of modulation.

Modulation voltage in this work is limited by area of linear control $\left(\left|\underline{U}^{*}\right|<\sqrt{3}\right)$, as overmodulation mode is normally not used in VSI of high quality AC drives.

\section{The model of inverter and assessment of the conversion quality}

To eliminate the influence of many secondary effects on the result the study was conducted in Matlab-Simulink on the model of idealized inverter (Fig. 6), each phase of which is a group of five ideal switches $\left(S_{A 1} \ldots S_{A 5}\right)$ with the switching algorithm corresponding in every moment to condition:

$$
\begin{aligned}
& S_{A 1}+S_{A 2}+S_{A 3}+S_{A 4}+S_{A 5}=1 ; \\
& S_{B 1}+S_{B 2}+S_{B 3}+S_{B 4}+S_{B 5}=1 ; \\
& S_{C 1}+S_{C 2}+S_{C 3}+S_{C 4}+S_{C 5}=1,
\end{aligned}
$$

where $S_{A 1} \ldots S_{A 5} ; S_{B 1} \ldots S_{B 5} ; S_{C 1} \ldots S_{C 5}$ are the switching functions of switches that take a value of one in the closed condition, and a value of zero - in opened.

The frequency of fundamental harmonic $f_{1}$ is used as the base unit in the model, of which relative modulation frequency $f_{c}$ and electromagnetic time constant of RL load $\tau_{R L}$ are:

$$
\begin{gathered}
f_{c}=6 n f_{1} \\
\tau_{R L}=R / L=1 /\left(5 f_{1}\right),
\end{gathered}
$$

where $n$ is a natural number.

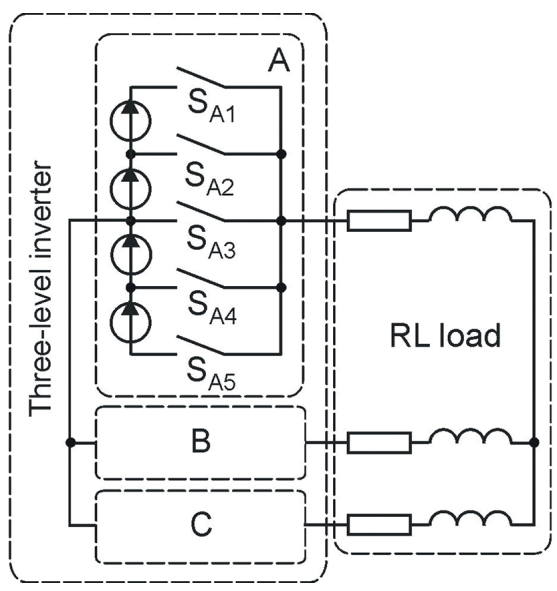

Fig. 6. The functional diagram of three-level inverter
Usually the conversion quality is assessed by total harmonic distortion coefficient of phase current:

$$
\mathrm{THD}=\frac{\sqrt{\sum_{k=2}^{\infty} I_{k}^{2}}}{I_{1}}
$$

but in drive system VSI must form circular hodograph of current vector, so ripples of current vector magnitude $|\underline{I}|$ should be measured. It could be done by coefficient of variation:

$$
\mathrm{CV}=\frac{\sqrt{\frac{1}{T_{1}} \int_{0}^{T_{1}}(|\underline{I}|(t)-\bar{I} \mid)^{2} d t}}{|\bar{I}|}
$$

where $\overline{\mid I}\left|=\frac{1}{T_{1}} \int_{0}^{T_{1}}\right| \underline{I} \mid(t) d t$ is the average value of current vector magnitude at period of fundamental harmonic $T_{1}$.

It could be shown that for the same phase current spectra and current vector magnitude THD and CV relates as $T H D / C V=\sqrt{2}$. But these spectra are always different.

Let the current magnitude contain, for example, odd high harmonics, i.e.

$$
|\underline{I}|=I_{0}+\sum_{k=3,5,7} I_{m k} \sin \left(k \omega t+\beta_{k}\right)
$$

Then the current vector turning with frequency $\omega$ is equal

$$
\underline{I}=\left[I_{0}+\sum_{k=3,5,7} I_{m k} \sin \left(k \omega t+\beta_{k}\right)\right] e^{j \omega t},
$$

and its projection to real axis, i.e. current of phase A, is

$$
\begin{aligned}
\operatorname{Re}(\underline{I}) & =i_{A}(t)= \\
= & {\left[I_{0}+\sum_{k=3,5,7} I_{m k} \sin \left(k \omega t+\beta_{k}\right)\right] \cos (\omega t)=} \\
= & I_{0} \cos (\omega t)+\sum_{k=3,5,7} I_{m k} \sin \left(k \omega t+\beta_{k}\right) \cos (\omega t)= \\
= & I_{0} \cos (\omega t)+\sum_{k=3,5,7} \frac{I_{m k}}{2}\left\{\begin{array}{l}
\sin \left[(k+1) \omega t+\beta_{k}\right]+ \\
+\sin \left[(k-1) \omega t+\beta_{k}\right]
\end{array}\right\} .
\end{aligned}
$$

As an example, in Fig. 7 higher harmonics of the current vector magnitude (a) and phase current (b) are shown for one of operation modes of the inverter.

For a complete picture of interdependence of THD and CV, differences between these coefficients were evaluated for all possible operation modes of the inverter. In Fig. 7(c) graphs of correlation coefficient of THD and CV are shown for different 
a)

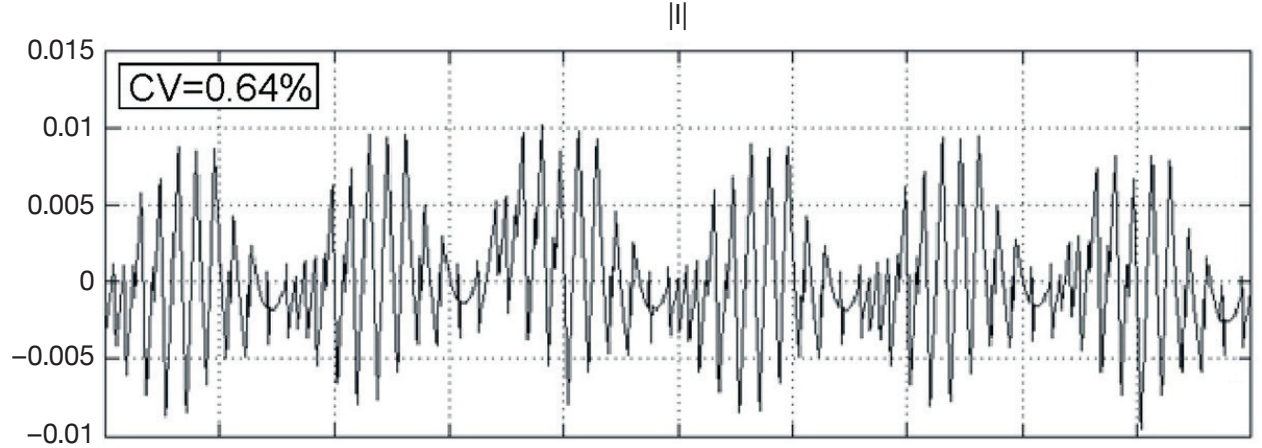

b)

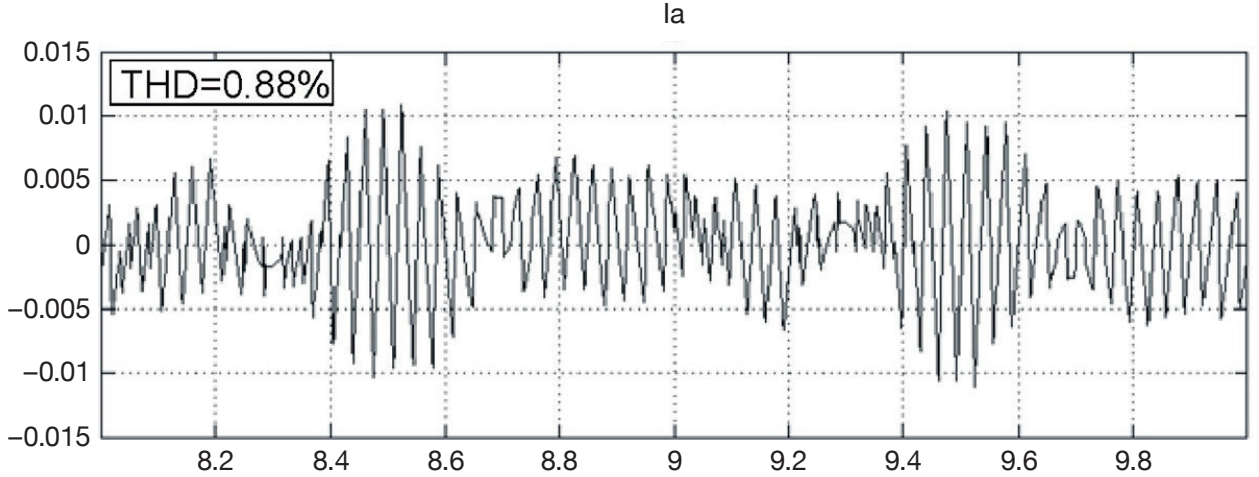

c)

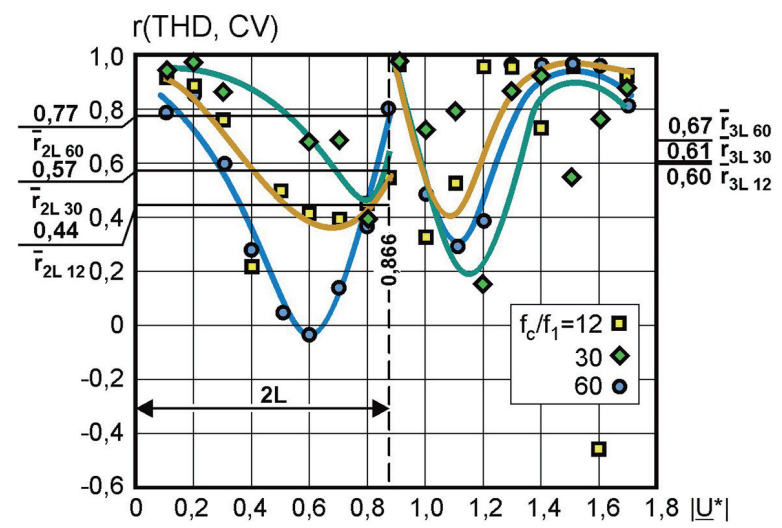

Fig. 7. The ripples of current vector magnitude (a) and its phase projection (b); correlation coefficient between THD of phase current and CV of current vector magnitude

levels of $\left|\underline{U}^{*}\right|$. Correlation coefficients have very low values even for middle levels of $\left|\underline{U}^{*}\right|$ in two-level $(\bar{r} 2 L 60, \bar{r} 2 L 30$ and $\bar{r} 2 L 12)$ and three-level $(\bar{r} 3 L 60, \bar{r} 3 L 30$ and $\bar{r} 3 L 12)$ operation modes, and for some values of $\left|\underline{U}^{*}\right|$ are close to zero and even anticorrelating between each other. Consequently, THD of phase current cannot be used to estimate the circular hodograph distortions.

\section{The research results}

Areas of possible values of CV are shown in Fig. 8(a) for different modulation frequencies and algorithms in steady state mode. These data show that peak-to-peak values relating to change of modulation algorithm, are 3.25, 3.9 and 2.4 for mul- tiplicity of modulation frequency 60,30 and 12 , respectively. There is no clear regularity in the distribution of minimum and maximum values of $\mathrm{CV}$ across modulation levels $\left|\underline{U}^{*}\right|$. Borders of areas and the mean value of $\mathrm{CV}$ have relatively monotonous character for two-level operating mode of the inverter. The borders have local extrema for three-level operation mode for all modulation frequencies. Special type of relation $\mathrm{CV}=f\left(\left|\underline{U}^{*}\right|\right)$ takes place in the transition area of modulation. At its lower border, all the functions $\mathrm{CV}=f\left(\left|\underline{U}^{*}\right|\right)$ have discontinuity, and at upper - local minimum.

The transition area possess interest also for analysis of the effect of modulation algorithms on the magnitude of $\mathrm{CV}$ ripples during A-V-A segment change. In Fig. 8(a) CV surfaces are shown for all possible combinations of algorithms in segments. From this figure and low values of the correlation coefficients between 
a)

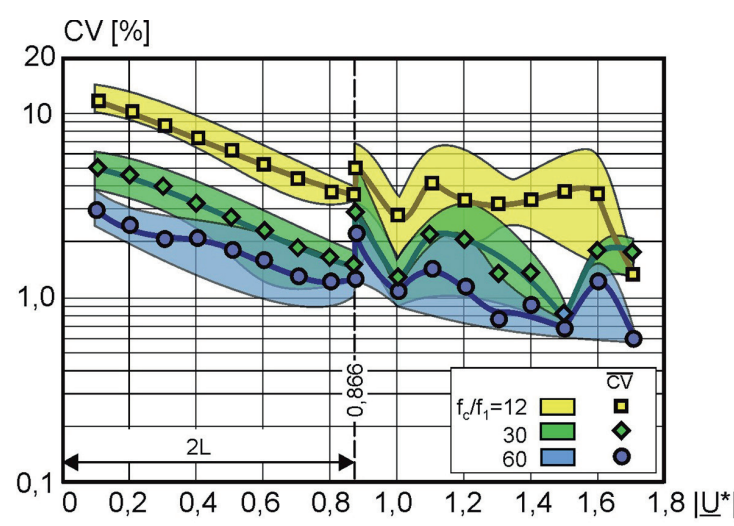

b)

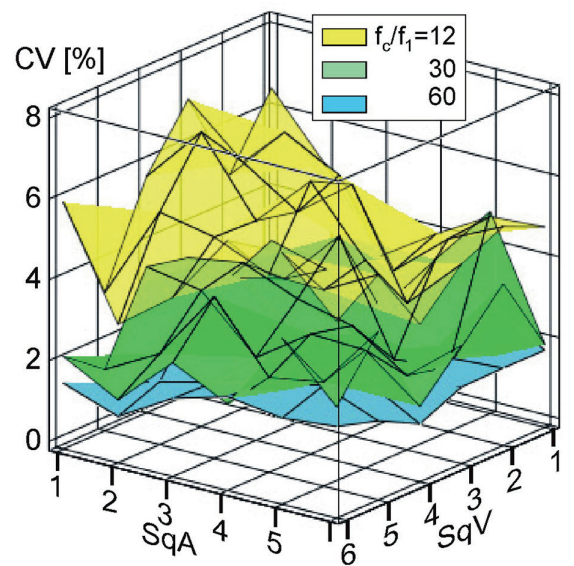

Fig. 8. Areas of current vector CV for different modulation algorithms and frequencies (a), and surfaces of CV for different modulation algorithms in $\mathrm{A}$ and $\mathrm{V}$ segments of the transition area of modulation (b)

a)

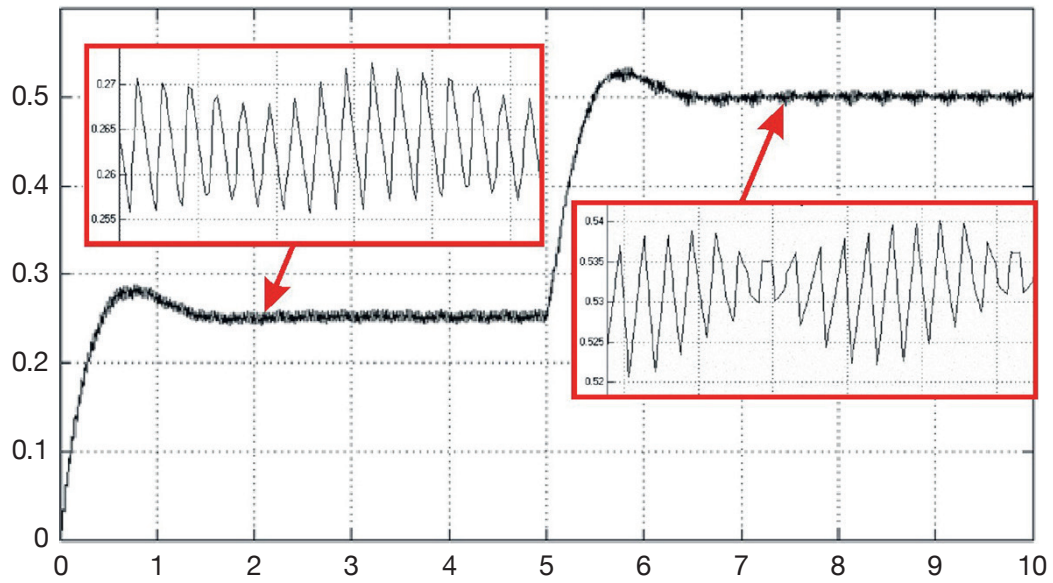

b)

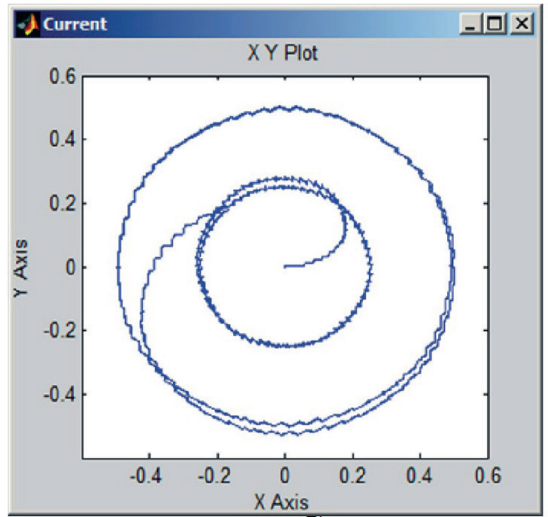

c)

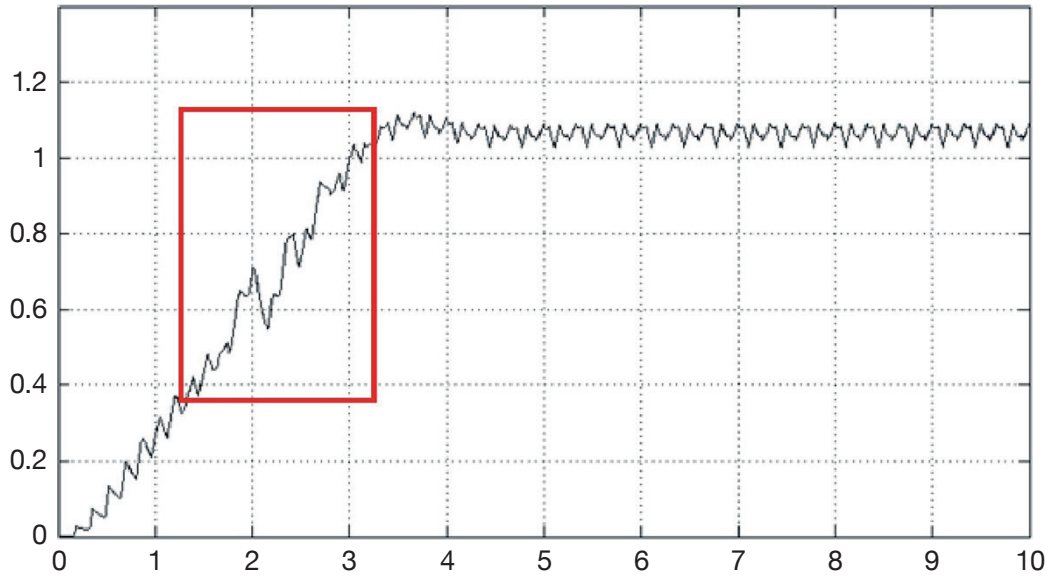

Fig.9. The magnitude of current vector (a) and its hodograph (b) for jump of $\left|\underline{U}^{*}\right|$ in the first area of modulation; the magnitude of current vector for linear increasing $\left|\underline{U}^{*}\right|$

points of surfaces (which do not exceed 0.5) it follows that there is no combination of modulation algorithms in $\mathrm{A}$ and $\mathrm{V}$ segments, providing minimum value of $\mathrm{CV}$ for all modulation frequencies.

The strong dependence of current ripples from modulation voltage leads to essential changing of conversion quality in the process of regulation. The response to stepwise change of $\left|\underline{U}^{*}\right|$ is shown in Fig. 9(a, b), where one can clearly see that ripples change in kind after the transition to the next value of current magnitude, which can lead to the self-oscillation excitation in the drive.

Self-oscillations can also arise in the process of smooth current control at the entrance to the transition area of modulation, which is associated with the stepwise change of spectrum of 
current magnitude at this point. Figure $9(\mathrm{c})$ shows the disturbance of the transient response in the transition area of modulation for a linear increase of modulation voltage.

\section{Conclusions}

1) Stepwise change of magnitude of load current ripples occurs in all multilevel voltage inverters at transitions from one modulation level to another, which is unacceptable for high quality drives working, for example, in tracking mode. The relative magnitude of jumps decreases with increase of modulation level, but for deep regulation mode bounds of current ripples and jumps will be determined by two and three-level operation modes of the inverter.

2) The magnitude and character of load current ripples can be significantly reduced by changing the modulation algorithm, but for optimizing operation of the inverter in wide regulation range, this change must be implemented in functional dependence on modulation voltage.

3) To maintain the voltage and current spectrum while changing the rotational direction of modulation vector, it is necessary to change the modulation algorithm to mirror symmetric one.

4) The magnitude of current hodograph distortion must be estimated by coefficient of variation, evaluated by straighforward calculation of magnitude.

Acknowledgement. This research was financially supported by government of Russian Federation, Grant 074-U01.

\section{REFERENCES}

[1] T.A. Glazenko and V.S. Tomasov, "Status and prospects of semiconductor converters implementation in instrument engineering", Izv.vusov. Priborostroenie 39 (6), 5-10 (1996).

[2] V.N. Vasiliev, V.S. Tomasov, M.A. Sadovnikov, and V.D. Shargorodsky, "Precision electric drive systems used in high accuracy complexes of watching for space objects, current statement and development prospects", Izv.vusov. Priborostroenie 51 (6), 5-12 (2008).

[3] M.A. Sadovnikov, V.S. Tomasov, and V.A. Tolmachev, "Precision electric drive for optical space control systems", Izv.vusov. Priborostroenie 54 (6), 81-86 (2011).

[4] K.E. Mikheev and V.S. Tomasov, "Analysis of energy performance of multilevel semiconductor converters of electric drive systems", Scientific and Technical Journal of Information Technologies, Mechanics and Optics 12 (1), 46-52 (2012).

[5] D.G. Sadikov, V.G. Titov, and R.E. Alexeev, "Development of optimized control algorithm for cascaded multi-level inverter", Proceedings of the IX International Conference on Power Drives Systems, Perm, 292-295 (2016).
[6] I.P. Voronin, P.A. Voronin, D.V. Rozhkov, and Y.T. Portnoy, "Problems of practical application of a three-level neutral-point clamped inverter circuit", Proceedings of the IX International Conference on Power Drives Systems, Perm, 266-269 (2016).

[7] P. Wójcik, D. Swierczyński, M. Kazmierkowski, and M. Janaszek, "Direct torque controlled PWM inverter fed induction motor drive for city transportation", Prace naukowe Institutu Maszyn, Napędów i Pomiarów Elektrycznych Politechniki Wrocławskiej 62/2008, 355-361 (2008).

[8] D. Swierczyński and M. Zelechowski, "Universal structure of direct torque control for AC motor drives", Przegląd Elektrotechniczny 5/2004, 489-492 (2004).

[9] G.S. Buja and M. Kazmierkowski, "Direct torque control of PWM inverter-fed AC motors - a survey", IEEE Transactions on Industrial Electronics 51 (4), 744-757 (2004).

[10] M. Malinowski, S. Styński, W. Kolomyjski, and M. Kazmierkowski, "Control of three-level PWM converter applied to variable-speed-type turbines", IEEE Transactions on Industrial Electronics 56 (1), 69-77 (2009).

[11] V.S. Tomasov and A.A. Usoltsev, "Comparative analysis of the energy efficiency of the scalar and spacevector PWM in a threephase inverter", Russian Electrical Engineering 85 (2), 111-114 (2014).

[12] P. Wiatr and M. Kazmierkowski, "Model predictive control of multilevel cascaded converter with boosting capability - a simulation study", Bull. Pol. Ac.: Tech. 64 (3), 581-590 (2016).

[13] H. Abu-Rub, D. Stando, and M. Kazmierkowski, "Simple speed sensorless DTC-SVM scheme for induction motor drives", Bull. Pol. Ac.: Tech. 61 (2), 301-307 (2013).

[14] M. Zygmanowski, B. Grzesik, M. Fulczyk, and R. Nalepa, "Selected aspects of modular multilevel converter operation", Bull. Pol. Ac.: Tech. 62 (2), 375-385 (2014).

[15] G. Buja, R. Menis, and R. Keshri, "Vector analysis of the current commutation in PM BLDC drives", Bull. Pol. Ac.: Tech. 61 (4), 829-836 (2013).

[16] D. Casadei, F. Profumo, G. Serra, and A. Tani, "FOC and DTC: Two viable schemes for induction motors torque control", IEEE Trans. Power. Electron. 17, 779-787 (2002).

[17] P. Chintan, P.P. Rajeevan, A. Dey, R. Ramchand, K. Gopakumar, and M. Kazmierkowski, "Fast direct torque control of an open-end induction motor drive using 12-sided polygonal voltage space vectors", IEEE Transactions on Power Electronics 27 (1), 400-410 (2012).

[18] J. Mathew, K. Mathew, N.A. Azeez, and K. Gopakumar, “A hybrid multilevel inverter system based on dodecagonal space vectors for medium voltage IM drives", IEEE Trans. Power Electron. 28 (8), 3723-3732, (2013).

[19] B.P. McGrath and D.G. Holmes, T. Lipo, "Optimized space vector switching sequences for multilevel inverters", IEEE Trans. Power Electron. 18 (6), 1293-1301 (2003).

[20] D.G. Holmes, "The general relationship between regular-sampled pulse-width-modulation and space vector modulation for hard switched converters", Conf. Rec. IEEE-IAS Annual Meeting, 1002-1010 (1992). 